\section{Afrontando con el alumnado la evaluación online de una didáctica del medio natural en tiempos de la COVID-19}

\section{Antonio Torralba-Burrial}

Universidad de Oviedo
Facing with the students the online evaluation of a didactics of the natural environment in times of COVID-19

\section{Abstract}

The COVID-19 pandemic has produced a global change situation in society, which has been confined and the teaching-learning processes at all educational levels have been restructured. The situation has been analysed with the students of the course of Knowledge of the natural and cultural environment, in the Degree in Early Childhood Education, with the aim to design an online evaluation process that allows a correct assessment of the competences and learning outcomes, suitable with the general and particular situation of the students. The different pattern of online access to the course is analysed, compared to the previous year, and the perceptions of the students about their situation and the results achieved during the course, as well as the students problems and possible solutions for a suitable online evaluation.

Key words: Teacher Education. Blended learning. Evaluation, Science Education.

\section{Introducción}

$\mathrm{Al}$ abordar una didáctica específica sobre ciencias experimentales en la formación inicial de los futuros maestros de Educación Infantil, como es el caso del conocimiento del entorno natural, hay que tener en cuenta tanto los conceptos básicos de alfabetización científica que deberían tener los docentes (incluso como parte de la ciudadanía universitaria) como las especificidades metodológicas y conceptuales de la etapa educativa en la que desarrollarán su profesión, siendo ambas cuestiones incluidas habitualmente en los manuales universitarios de la materia actuales (p.ej., Fernández \& Bravo, 2015; Mérida, Torres-Porras \& Alcántara, 2017; Quijano, 2016). Se trata de la enseñanza de una ciencia que va a tener que potenciar habilidades propias del trabajo científico (de observación/clasificación de objetos y situaciones, razonamiento, capacidad de pasar de las observaciones a las conclusiones), unos contenidos (correspondientes con las tres áreas del currículo de Ed. Infantil, si bien más ampliamente recogidos en el conocimiento del entorno) y la búsqueda de estrategias para enseñarlos en Ed. Infantil (Cantó, de Pro \& Solbes, 2016). 
Referencias a estas cuestiones vienen incluidas en las guías docentes de las asignaturas relacionadas con la didáctica de las ciencias experimentales asociadas a la adquisición de las correspondientes competencias oficiales en el Grado en Maestro en Educación Infantil en España, si bien de una forma no homogénea, siendo también evaluadas con diferentes criterios e instrumentos, principalmente traducidos en un examen y la elaboración de trabajos y actividades más prácticas (de Pro Bueno, de Pro \& Cantó, 2019). De estas actividades prácticas, las más frecuentes en los planes de formación de maestros son las relacionadas con con la indagación, y suelen estar menos representadas las de modelización y las de argumentación, en ocasiones escasamente (Bargiela, Puig \& Blanco, 2018).

Esas cuestiones fueron tenidas en cuenta en el diseño de la asignatura de Conocimiento del entorno natural y cultural en la Universidad de Oviedo, que se ha ido implementando en los últimos años siguiendo criterios del aprendizaje mixto: clases expositivas, prácticas de aula y de laboratorio presenciales, si bien con apoyo, materiales, recursos didácticos y entregas de trabajos, porfolios y cuestionarios a través del aula virtual de la asignatura. Y, como en la mayoría de las asignaturas, una prueba final presencial consistente en un examen escrito.

Sin embargo, el virus SARS-CoV-2 provocó la pandemia de alcance mundial de la COVID-19, alcanzando a España en el segundo semestre del curso 2019/20. Entre el 11 y el 14 de marzo de 2020 se produjo la declaración de pandemia mundial por parte de la Organización Mundial de la Salud (OMS), los cierres presenciales de todos los centros educativos en el país y la declaración del estado de alarma en España. Al principio, sin un planteamiento claro de cuánto tiempo iba a durar la situación, se continuó con la docencia en línea en las asignaturas que seguían activas y se atendió a distancia al alumnado. García-Peñalvo, Corell, Abella-García \& Grande (2020) han realizado un análisis de situaciones y recomendaciones para el seguimiento de las asignaturas y evaluación en línea en las enseñanzas universitarias, según los distintos escenarios esperables en esta situación, de gran utilidad para comenzar a reflexionar sobre qué se busca con la evaluación y posibles formas de conseguirlo. La pandemia y el confinamiento han generado un enorme estrés en el sistema educativo, con una disrupción que ha descolocado tanto al alumnado como al profesorado en todo el mundo (IESALC, 2020). Y, pese a la situación de cierre presencial, se han aplicado estrategias, metodologías y alternativas en línea para mitigar dicha disrupción en todo el mundo (p.ej., Ferdig,
Baumgartner, Hartshorne, Kaplan-Rakowski \& Mouza, 2020). En el caso de la Universidad de Oviedo, las indicaciones fueron claras sobre que el curso continuaría de forma telemática, a través del campus virtual y/o de los programas de MS Office 365 , y que se debía trabajar en el escenario de una evaluación online salvo casos excepcionales.

Pero pasar de los planteamientos generales a la particularidad de cada asignatura no resulta automático, requiere repensar y reflexionar sobre la situación, el desarrollo de la docencia y las condiciones de evaluación, alejadas de cualquier óptimo. Cuestiones como la brecha digital, que afecta diferencialmente al alumnado en su acceso a los servicios educativos telemáticos que requieran banda ancha (Cabero \& Ruiz, 2018; Gómez, 2019), los espacios en los que profesorado y alumnado realizan los procesos de enseñanza-aprendizaje, las situaciones laborales, familiares y psicológicas derivadas de la pandemia (Lozano-Díaz, Fernández-Prados, Figueredo Canosa \& Martínez Martínez, 2020), se convierten en variables clave a conjugar con las competencias y resultados de aprendizaje a la hora de plantear esta adaptación acelerada de la evaluación docente, no solo pero también en la educación superior (Echeita, 2020).

Y quien mejor puede conocer y valorar cuál es su situación real, o por lo menos como esta situación es percibida, es el propio alumnado.

Todo ello, sin perder de vista que se trata de una evaluación a maestros en formación, por lo que el sistema de evaluación debería poder valorar sus capacidades de diseño de procesos de enseñanza-aprendizaje, en los que los conocimientos científicos estén acompañados de las metodologías para enseñarlos en su etapa educativa (Smith, 2016).

\section{Objetivos}

El objetivo de este artículo es exponer los resultados obtenidos y las reflexiones sobre la evaluación en línea en una didáctica del entorno natural orientada a la formación inicial de maestros de Educación Infantil.

Para poder valorar en qué medida es afectado el estudiantado por esta situación, desde una perspectiva de seguimiento y finalización del curso y de valoración de medidas mitigadoras de dicha problemática en la evaluación en línea, se optó por 
una aproximación directa mediante cuestionario de autopercepciones dirigido al alumnado, complementado con el análisis de los datos de actividad en el aula virtual de la asignatura.

\section{Metodología}

\section{Contexto}

La experiencia se ha llevado a cabo en la asignatura de Conocimiento del entorno natural y cultural, asignatura obligatoria anual que se cursa en $3^{\circ}$ del Grado en Maestro en Educación Infantil, en la Facultad de Formación del Profesorado y Educación de la Universidad de Oviedo. Se ha aplicado en el grupo de tardes, con un alumnado matriculado de 56 personas, de las cuales 54 han interaccionado de alguna manera con la asignatura durante el curso. La asignatura se organiza atendiendo a un aprendizaje mixto: formación presencial acompañada de materiales, actividades y recursos a través del aula virtual, alojada en el campus virtual de la Universidad en la plataforma Moodle v3.0. Para la generación de dichos materiales didácticos, y en gran medida para la selección, se han seguido criterios de infoaccesibilidad (Torralba-Burrial \& Herrero, 2018). La asistencia a los distintos tipos de actividades es alta (Figura 1), en especial a las prácticas de laboratorio y aula (85-93\% del alumnado que contesta al cuestionario afirma haber asistido presencialmente a más del $75 \%$ de las mismas), seguidas de las clases presenciales (con la mitad del alumnado habiendo asistido a más de la mitad de las clases, si bien solo un 20\% afirman haber asistido a más del $75 \%$ ) (un 13\% de las personas que han contestado están matriculados con evaluación diferenciada o segunda y posteriores matrículas asistiendo parte a las prácticas de laboratorio y aula).

La situación sanitaria derivada de la pandemia de la COVID-19 representó la suspensión de la docencia presencial en la Universidad de Oviedo el 13 de marzo de 2020, habiendo finalizado (de acuerdo con el calendario docente habitual) las clases expositivas, prácticas de aula y de laboratorio de la asignatura esa semana.
A) Tipología seguimiento

B) Seguimiento presencial autopercibido alumnado
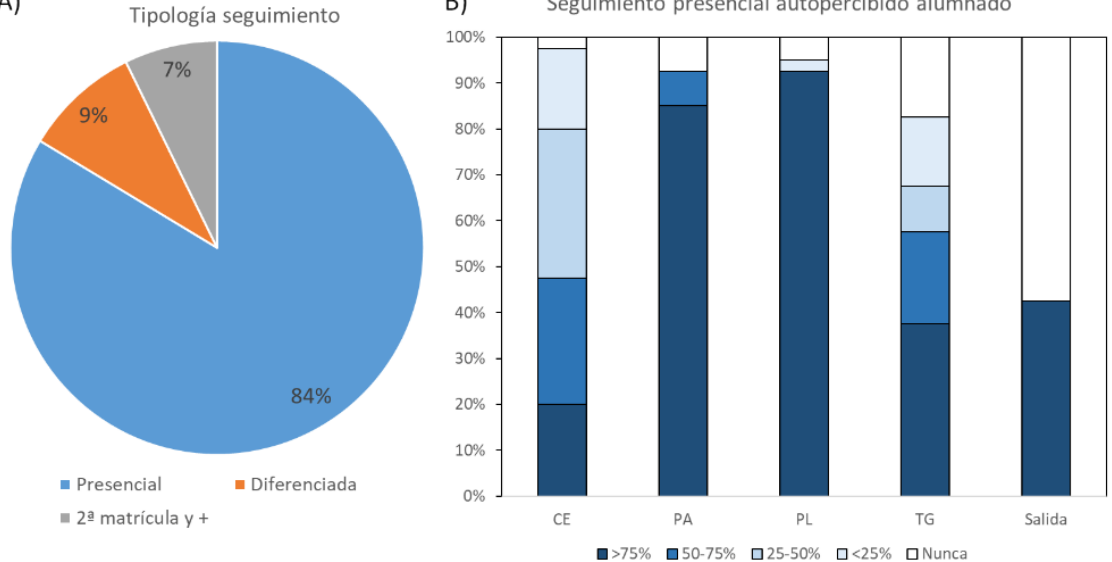

Figura 1. Seguimiento de la asignatura por el alumnado, curso 2019/20. A) Tipología según matrícula oficial $(n=56)$. B) Autopercepción del seguimiento presencial por parte del alumnado que contestó al cuestionario $(n=40)$. CE = clases expositivas; $\mathrm{PA}=$ prácticas de aula; $\mathrm{PL}=$ prácticas de laboratorio; $\mathrm{TG}=$ tutoría grupal; Salida $=$ salida didáctica voluntaria de día entero.

\section{Análisis de actividad en el aula virtual}

Se ha realizado una extracción de los registros de actividad en el aula virtual del grupo de tardes de la asignatura, tanto del curso 2018/19 (20 133 registros) como del curso 2019/20 (24 522 registros). De la base de datos generada (44 655 registros), se han eliminado los datos referentes a la actividad del profesorado y actividades administrativas de los cursos, manejando los datos con el programa MS Excel para Microsoft 365. El análisis se ha realizado mediante las funciones de tablas dinámicas.

\section{Autopercepción situación alumnado}

Un cuestionario en línea con 26 preguntas fue diseñado empleando Microsoft Forms y accesible a través del aula virtual de la asignatura. El sistema garantizaba que cada estudiante solo contestara una vez y que quedara registrada su respuesta asociada a su identificación universitaria, facilitando el poder facilitar soluciones particulares para casos particulares si era necesario. Se incluyeron 26 ítems para valorar las 
percepciones (utilizando una escala tipo Likert de cinco niveles cuando fue apropiado) y preguntas abiertas para recoger el espectro más amplio de situaciones y problemáticas.

Una primera sección del cuestionario, de dos preguntas, buscaba clasificar cómo había seguido el alumnado la asignatura y en qué medida había asistido a las diferentes experiencias formativas, conformando el contexto real de la asignatura que podríamos considerar periodo pre-COVID-19 (Figura 1).

Una segunda sección buscaba valorar la autopercepción de la medida en la que cada alumno había adquirido las competencias específicas de la materia y alcanzado los resultados específicos de aprendizaje, así como una autoevaluación en la asignatura atendiendo a los dos puntos anteriores y a su desempeño en la asignatura. El alumnado disponía en el momento del cuestionario de las calificaciones de las prácticas de aula realizadas.

La sección final buscaba recoger la información sobre las repercusiones académicas de la situación en la que se encontraban, derivada de la pandemia de COVID-19, mediante preguntas abiertas (problemas específicos para realizar el examen en línea y posibles soluciones que consideraban pudieran solucionarlos), así como preguntas de percepción sobre en qué medida podrían paliarse o solucionarse esos problemas adoptando alguna de las medidas propuestas desde el profesorado.

\section{Resultados y discusión}

\section{Situación durante la COVID-19}

La suspensión de las clases presenciales en la Universidad de Oviedo ocurrió el 13 de marzo, indicándose el día 12. Dicha semana se terminaban, de acuerdo con el calendario habitual de la asignatura, las clases expositivas, las prácticas de aula y de laboratorio. La relación con el alumnado se mantuvo a través de herramientas asíncronas (correo electrónico, foros del aula virtual de la asignatura) y síncronas (tutorías por MS TEAMS a solicitud alumnado).

La primera cuestión que valorar a la hora de definir las actuaciones en periodo de no presencialidad marcada por la COVID-19, tanto el seguimiento de ese último tramo de la asignatura como la evaluación, era valorar si el alumnado seguía manteniendo en sus nuevas localizaciones la capacidad de conexión al campus virtual. De hecho, se comprobó que el $90 \%$ del alumnado que había entrado alguna vez en el aula virtual de la asignatura accedió a la misma, igualmente, en algún momento de la semana del 6 al 12 de abril.

Todo el alumnado que había asistido a las prácticas de laboratorio pudo entregar el correspondiente porfolio en la tarea habilitada para ello en el aula virtual, lo que indicó que disponían de la capacidad de conexión para manejar archivos de tamaño medio (varios archivos formato pdf de hasta $40 \mathrm{Mb}$, debido al acompañamiento gráfico de las prácticas), al menos de forma asíncrona, en la semana del 15 de abril, al mes de iniciarse el confinamiento.

En cuanto al seguimiento de los materiales, recursos, actividades y foros accesibles en el aula virtual de la asignatura, la comparación con los accesos del alumnado el curso anterior (Figura 2), muestra un incremento en los accesos en los meses de marzo y abril (periodo dedicado normalmente a las prácticas en los centros de Educación Infantil, incluido el curso 2018/19, y que se corresponde con el periodo que el alumnado ha estado confinado en el curso 2019-20), una disminución en mayo y un nuevo incremento en junio (valores comparativos alterados por la comunicación de calificaciones de la convocatoria ordinaria de la asignatura a finales de mayo en 2019 y a principios junio en 2020). En todo caso, muestra que, en términos generales, el alumnado accedía regularmente y en mayor medida que el curso anterior al aula virtual de la asignatura.

Esto resulta coherente con lo observado (pero no cuantificado) sobre el mayor interés del alumnado en tutorías en línea en el periodo de confinamiento que el curso pasado en tutorías presenciales en fechas equiparables. 


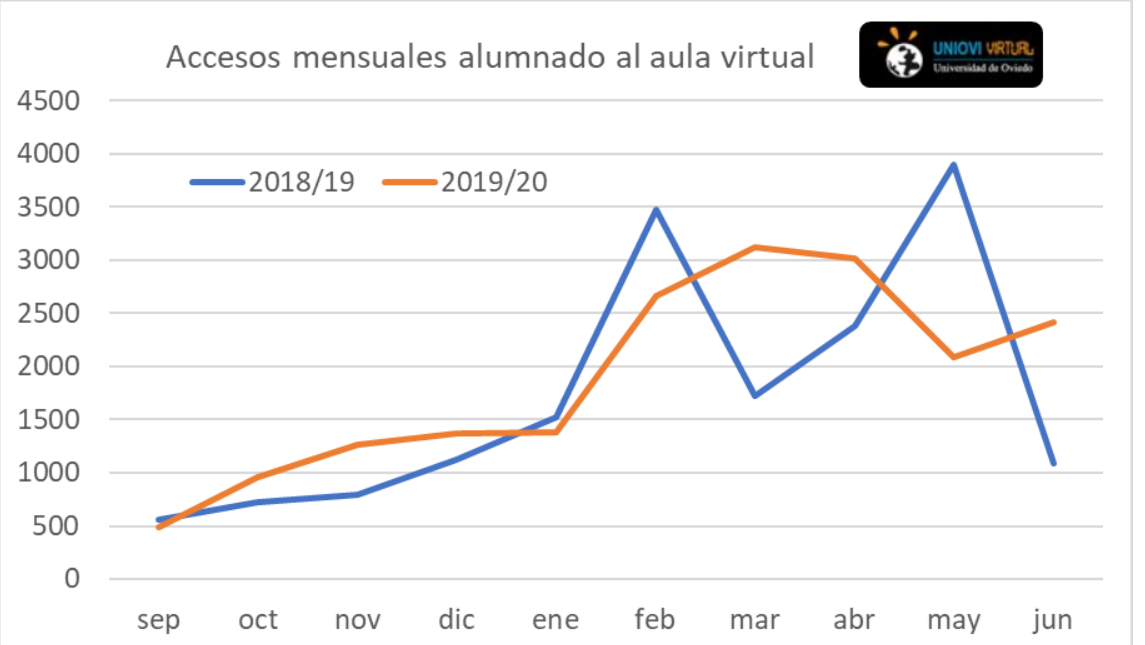

Figura 2. Comparativa de accesos mensuales (a foros, actividades, materiales o recursos didácticos) del alumnado al aula virtual de la asignatura entre el curso 2018/19 y el 2019/20.

\section{Autoevaluación del aprendizaje}

La autopercepción manifestada por el alumnado sobre el grado en el que habían desarrollado las competencias específicas de la materia asignadas a la asignatura hasta ese momento mostró unos resultados muy positivos (Figura 3). En primer lugar, fue realizada por una inmensa mayoría del alumnado $(71 \%$, que sube hasta el $82 \%$ si excluimos del total a aquellas personas que habían aprobado la asignatura en la convocatoria extraordinaria adelantada y a aquellas que no habían interaccionado con la asignatura hasta ese momento).

En segundo lugar, no hubo estudiantes que manifestaran un desarrollo nulo de ninguna de las competencias, mientras que fue muy escaso el porcentaje que manifestó en alguna competencia que consideraba haberla desarrollado poco. Las competencias que el alumnado indicó haber desarrollado en mayor medida fueron el conocimiento del método científico (y promover el pensamiento científico y la experimentación) (78\% la consideran desarrollada completamente o mucho), promover interés y respeto por el medio natural y cultural a través proyectos didácticos (93\%) y elaborar propuestas didácticas en relación con la interacción Ciencia, Tecnología, Sociedad y Desarrollo Sostenible (88\%).

\section{MAGISTER}

Vol. 32. Núm. 1: (2020). Sección extraordinaria
La percepción del alumnado sobre el grado de alcance de los resultados de aprendizaje propios de la asignatura (Figura 4) proporcionó resultados igualmente satisfactorios: 10 de los 13 resultados de aprendizaje esperados fueron percibidos como alcanzados en grado alto o completamente por más de dos tercios del alumnado, mientras que ningún estudiante manifestó un grado nulo de alcance de ningún resultado de aprendizaje, y únicamente el 15\% manifestó haber alcanzado un grado bajo el objetivo de fomentar experiencias con las Tecnologías de la Información y Comunicación en Educación Infantil (el objetivo con menor grado de alcance autopercibido)

Autopercepción desarrollo compentencias alumnado

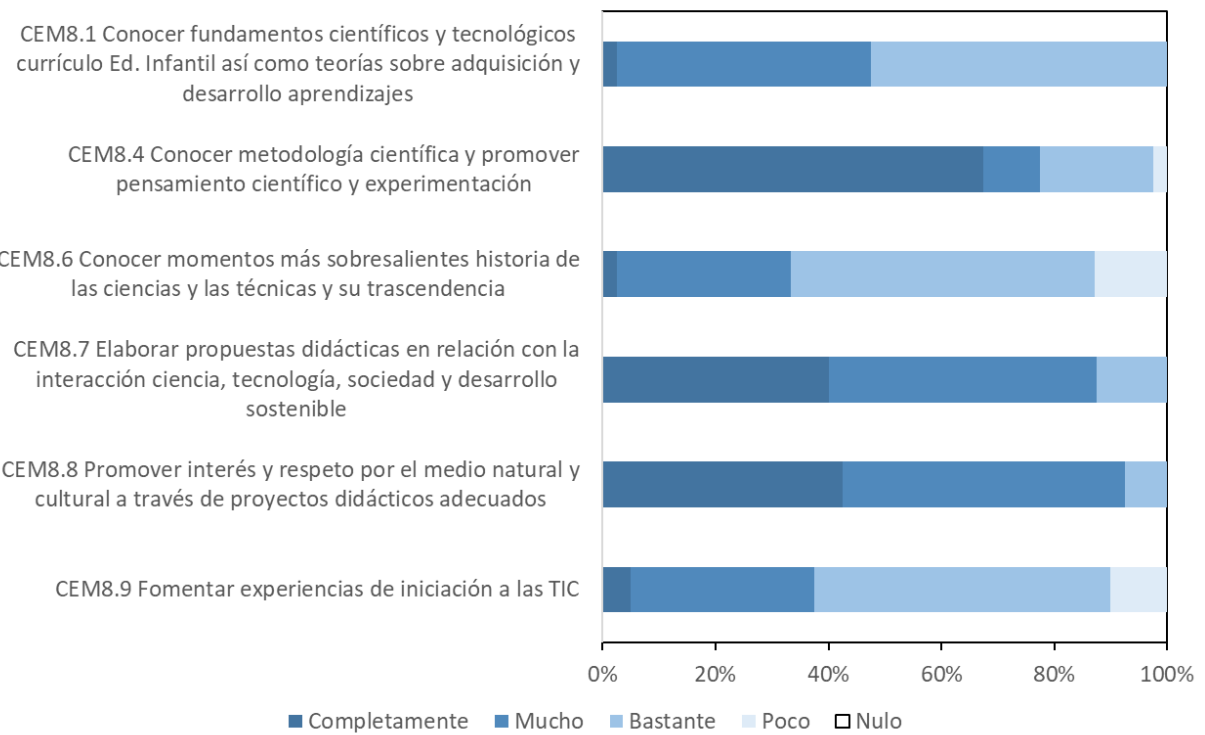

Figura 3. Autopercepción del grado de desarrollo de las competencias específicas de la materia por parte del alumnado hasta la interrupción de las clases presenciales $(n=40)$ 




Figura 4. Autopercepción sobre el grado de alcance de los resultados de aprendizaje propios de la asignatura por parte del alumnado hasta la interrupción de las clases presenciales $(n=40)$.

Aunque es cierto que una autopercepción puede ser (bastante) diferente de una evaluación objetiva, a efectos de elaborar la estrategia para la finalización del curso de forma no presencial, reviste utilidad que el alumnado perciba que el proceso de enseñanza-aprendizaje llevado a cabo ha funcionado con éxito y han podido desarrollar las competencias y adquirir los resultados de aprendizaje esperados al diseñar la asignatura. En todo caso, una cierta calibración se puede estimar a partir de la pregunta sobre la calificación que creen obtendrían en la asignatura: un 58\% indicaron la misma calificación que obtuvieron realmente (un 38\% estimaron una diferencia de una categoría, un 5\% de dos categorías).

\section{Problemas percibidos y posibles soluciones para la evaluación en línea}

Los problemas percibidos por el alumnado a la hora de abordar la evaluación en línea de la asignatura son más variados que los derivados de mantener conexiones asíncronas para el seguimiento de la asignatura (Figura 5). Su principal preocupación, incluida en las tres cuartas partes de las respuestas obtenidas, derivaba de la posible falta de conexión puntual a lo largo del examen, ya fuera propia o del campus virtual de la Universidad. Y esta preocupación incluía tanto a alumnado que manifestaba tener problemas o pérdidas de conexión puntuales habitualmente, como a aquel que indicaba que no los tenía nunca. La siguiente preocupación tenía que ver con la comunicación profesorado-alumnado durante la realización de la evaluación en línea, atendiendo a la posible saturación del canal que se empleara, a la no sincronización de la comunicación, a la falta de conexión de datos del ordenador o a la posible falta de comunicación durante toda la prueba. El resto de los problemas percibidos ya no son tan generales, sino que se corresponden con situaciones concretas de estudiantes concretos: problemas con el equipo informático o con las condiciones de realización de la prueba (distracciones derivadas del entorno, conexión general peor que la habitual de residencia, diferentes habilidades para la escritura por ordenador frente a manual). Esto no quiere decir que sean problemas menos importantes a la hora de no dejar a ningún estudiante atrás por problemas derivados de la situación, sino que pueden ser más abordables al afectar a un número muy reducido de estudiantes que si afectan a su conjunto. 
Problemas percibidos alumnado

Indica que ningún problema Mayor lentitud/ortografía examen a ordenador Diferente (mayor) exigencia en evaluación Equipo informático antiguo Distracciones por lugar realización no adecuado Conexión deficiente lugar residencia no habitual

Resolución dudas durante el examen Pérdida de conexión durante el examen

$$
\begin{array}{llllllll}
0 & 5 & 10 & 15 & 20 & 25 & 30 & 35
\end{array}
$$

Número de respuestas que lo incluyen

Figura 5. Posibles problemas percibidos por el alumnado para afrontar la evaluación en línea de la asignatura mediante un examen $(n=35)$.

A la hora de proponer posibles soluciones para solucionar los problemas que presentaban como estudiantes (Figura 6), las opciones más repetidas fueron no realizar examen (bien porque se sustituyera por una tarea o trabajo a entregar un día o varios después de ser enunciado, bien porque se recurriera exclusivamente a las calificaciones obtenidas en la evaluación continua) o añadir el suficiente tiempo al desarrollo de la prueba para asegurar que no había problemas con la entrega del examen. Poder hacer alguna prueba para comprobar que se podía desarrollar el examen adecuadamente, o facilitar una plataforma o sistema alternativo para la entrega del examen fueron también opciones generales, correspondiéndose con cierto temor al fallo del campus virtual de la Universidad.

\section{MAGISTER}

Vol. 32. Núm. I: (2020). Sección extraordinaria
Soluciones consideradas por alumnado

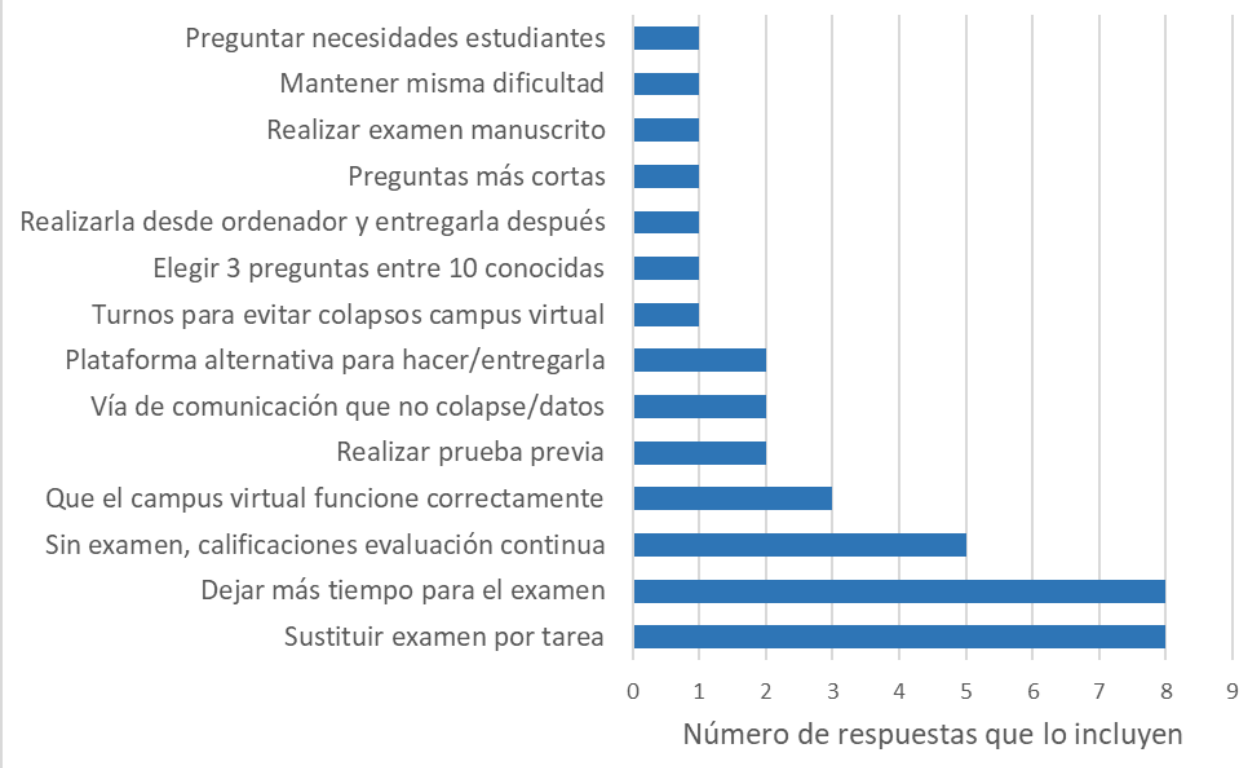

Figura 6. Posibles soluciones consideradas por el alumnado para mitigar los problemas percibidos (n $=32$ ).

Las posibles medidas propuestas por el profesorado fueron dirigidas a facilitar una experiencia lo más similar posible al examen presencial y buscar una complementación a la entrega a través del campus virtual por si fallaba este. En cuanto a garantizar la comunicación síncrona durante el examen, que era otro de temores expresados por el alumnado, se propusieron diversas vías de comunicación, basadas en la plataforma del campus virtual, externas a dicha plataforma e incluso sin necesidad de datos en el ordenador o el móvil, para cubrir los posibles escenarios durante la realización de la prueba en línea. El análisis visual de las percepciones del alumnado ante dichas opciones muestra que ninguna resulta por si misma completamente satisfactoria para todo el alumnado (Figura 7). No obstante, mantener la misma estructura del examen, realizándolo manuscrito y entregándolo fotografiado, fue considerado que mitigaba sus problemas entre bastante y completamente para el $63 \%$ (si bien para el $45 \%$ solo lo mitigaba bastante) y la posibilidad de contar con una segunda vía de entrega del examen (correo 
electrónico) permitía subir el porcentaje de alumnado que consideraba mitigado el problema hasta el $81 \%$. En el caso de la comunicación síncrona con el alumnado, la habilitación de un foro específico en el campus virtual y la posibilidad de comunicarse mediante WhatsApp fueron las mejor valoradas individualmente, mientras que las menos valoradas fueron la implementación de la mensajería instantánea del campus virtual y la posibilidad de comunicación mediante mensajes cortos por teléfono (SMS). Al valorar la complementariedad entre lo indicado por el alumnado para las distintas opciones, se encontraron los valores más altos de mitigación percibida si se unían todas las opciones propuestas menos la mensajería, alcanzándose un $92 \%$ de alumnado con mitigación percibida entre bastante y completa con alguna/varias de esas medidas (Figura 7).

¿En qué medida se mitigarían tus problemas si hubiera...?

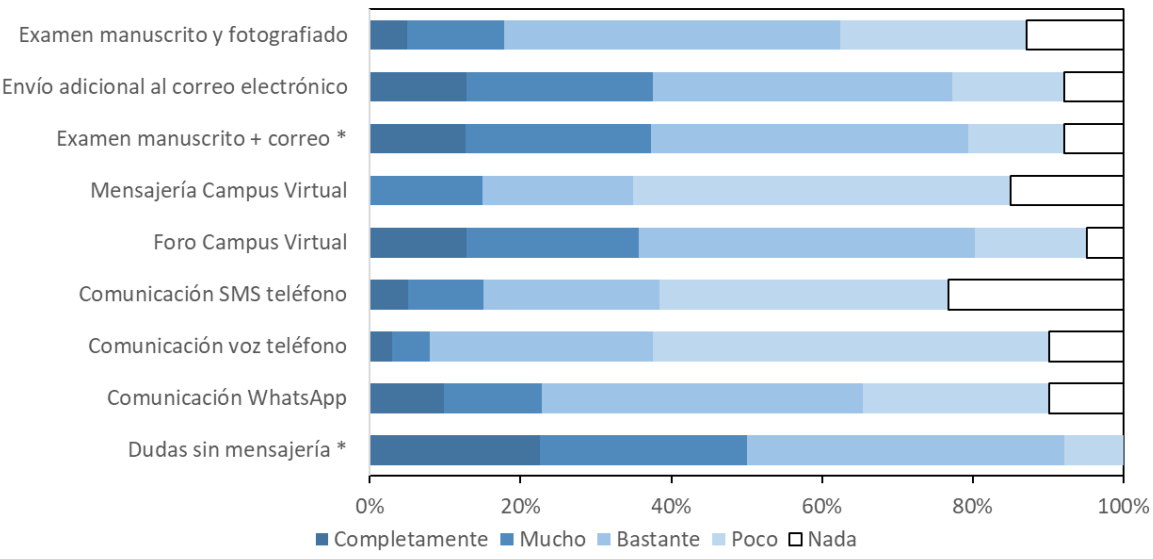

Figura 7. Valoración por parte del alumnado del grado en que serían mitigados sus problemas frente a la evaluación en línea si se aplicaran algunas medidas indicadas por el profesorado para facilitar desarrollo/entrega examen y resolución de dudas durante el mismo $(n=40)$ * La valoración de las opciones con múltiples medidas ha sido construida a partir de las respuestas a las medidas que engloban, por lo que pueden quedar subestimadas.

\section{Diseño de la evaluación en línea}

Tras comprobar que el alumnado seguía teniendo conexión a Internet (aunque no fuera óptima en varios casos) que le permitía acceder al aula virtual, entregar tareas y consultar materiales y recursos didácticos, se decidió mantener el peso de las distintas partes de la asignatura en la calificación global: $20 \%$ para las prácticas de aula $(7$ grupales y 3 individuales, realizadas en el aula antes de la suspensión de la actividad presencial, y entregadas en el aula virtual, la última en plazo después del cierre presencial), 20\% para el porfolio de laboratorio (6-7 prácticas realizadas grupalmente pero con reflexiones y adaptaciones individuales) y $60 \%$ para la prueba final escrita en línea. El motivo principal de no realizar un cambio en las ponderaciones fue asumir la posibilidad de que el alumnado hubiera invertido un determinado esfuerzo para unas prácticas que valían un $20 \%$ de la calificación global, y que ese esfuerzo pudiera haber sido diferente si el peso de esos trabajos hubiera sido distinto. De esta forma, se ha podido respetar, en la medida de lo posible, la guía docente con la que el alumnado accedió a la asignatura. A ese respecto, hubo estudiantes que se comunicaron en sentido del mantenimiento y otros del cambio de ponderación.

Se mantuvo también la estructura inicialmente prevista para la prueba presencial (3 preguntas a desarrollar, que implican conocer la materia, implementarla mediante actividades y realizar reflexiones y valoraciones sobre la misma). Tratándose de una didáctica específica sobre medio natural orientada a las especiales características de la Educación Infantil, y teniendo en cuenta que 4 de las 6 competencias específicas de la materia y que 10 de los 13 resultados de aprendizaje previstos en la guía docente de la asignatura implican diseño y/o elaboración de actividades para los procesos de enseñanza-aprendizaje del entorno natural (además del conocimiento de los conceptos necesarios sobre medio natural), no se consideró que un examen de otro tipo (p.ej., un cuestionario tipo test o de preguntas cortas) pudiera dar una información equivalente sobre el grado de desarrollo de las competencias y la consecución de los resultados de aprendizaje requeridos en la asignatura. La prueba de evaluación mantiene el principio de coherencia con los niveles de aprendizaje expresados en los resultados de aprendizaje en la guía docente, sin caer en la disminución de nivel que afecta en ocasiones a la evaluación online (Dorrego, 2016). En esencia, se ha buscado una prueba de evaluación en la que los maestros en formación tuvieran que plantear la aplicación de sus conocimientos, científicos y didácticos, sobre el entorno natural a la resolución de cuestiones reales de su profesión (Sanmartí \& Marchán, 2015), aunque fuera dentro de una realidad virtual hipotética.
MAGISTER

Vol. 32. Núm. 1: (2020). Sección extraordinaria 
Con el fin de que la experiencia para el alumnado fuera lo más similar posible a la realización de la prueba presencial prevista inicialmente, y atendiendo a los comentarios expuestos por parte del alumnado en el cuestionario, se optó por realizar un examen manuscrito y su envío fotografiado/escaneado a través del campus virtual, empleando la actividad Tarea dentro de Moodle. La decisión de la Universidad de montar en servidores externos un segundo campus virtual específico para exámenes, con más capacidad y resistencia facilitó la confianza en que se pudiera realizar el examen sin problemas. De acuerdo con lo manifestado por el alumnado, y aunque el funcionamiento y aspecto del campus virtual temporal era muy similar al habitual (funcionaba bajo la plataforma Moodle v3.5), se habilitó una prueba para que pudiera ensayar a realizar fotografías con el móvil y entregarlas como tarea los días previos al examen en el nuevo entorno. En todo caso, se mantuvo el campus virtual habitual como plataforma de reserva por si ocurría algún problema durante el desarrollo de la prueba en el nuevo, y además se habilitó la posibilidad de envío por correo electrónico del examen en caso de que fallaran ambos.

Respecto a las posibilidades de solución de dudas y comunicación síncrona con el profesorado, otro de los problemas que se percibían como causantes de mayor intranquilidad en el alumnado, se tuvieron en cuenta las valoraciones realizadas en el cuestionario. Así, se habilitó: 1) un foro específico en el aula del campus virtual temporal para exámenes, con el fin de facilitar la comunicación; 2) el chat de equipo para toda la clase e individual en MS Teams; 3 ) la posibilidad de comunicación por teléfono con el profesorado (WhatsApp, voz y SMS). El alumnado empleó durante el desarrollo de la prueba fundamentalmente el chat de equipo de MS Teams para la solución de dudas y, ante problemas o temores de último momento sobre la carga de los archivos en la tarea, también el WhatsApp. Pese a la seguridad manifestada por el alumnado en que disponer del foro del campus virtual podría mitigar sus posibles problemas (Figura 7), únicamente una alumna lo empleó para buscar la resolución de dudas durante el examen. No fueron necesarias comunicaciones por teléfono por voz ni por SMS, puesto que ningún estudiante perdió la conexión durante el examen (al menos no durante los momentos en los que necesitó solucionar dudas o entregar el examen).

Las medidas encaminadas a una experiencia de la evaluación en línea más similar a la evaluación presencial, así como proporcionar vías alternativas de comunicación, facilita disminuir el nerviosismo ante la realización de la prueba en un ambiente diferente al habitual y en línea, lo que puede facilitar las actitudes del alumnado ante la evaluación en línea y sus resultados (Celik \& Yesilyurt, 2013).

La prueba de evaluación, al incorporar necesariamente en su realización distintas actividades diseñadas por el alumnado y reflexiones propias, resultaba menos propicia para conductas deshonestas, lo que parecía ser una preocupación entre el profesorado universitario sobre las evaluaciones en línea (Chirumamilla, Sindre \& Nguyen-Duc, 2020; García-Peñalvo et al., 2020). Tan solo tres exámenes seguían una similitud elevada en el orden de planteamiento en las actividades de cada pregunta, con un desarrollo algo diferente y reflexiones individuales distintas, por lo que no resultaban más similares que los exámenes entregados por el alumnado que había preparado conjuntamente la asignatura en pruebas equivalentes presenciales de cursos anteriores. Las calificaciones finales de la asignatura, ponderando la calificación de la prueba final con las de la evaluación continua, no mostraron grandes diferencias con las obtenidas por el alumnado del curso anterior (Figura 8), siendo las diferencias más notables una disminución del porcentaje de notables y un ligero incremento del de aprobados (sin disminuir el de suspensos). Las causas pueden tener que ver con la tensión adicional derivada de la situación de pandemia por la COVID-19 y la realización del examen en un entorno físico no académico y, por tanto, con más probabilidad de que se dieran distracciones durante la realización de la prueba. 


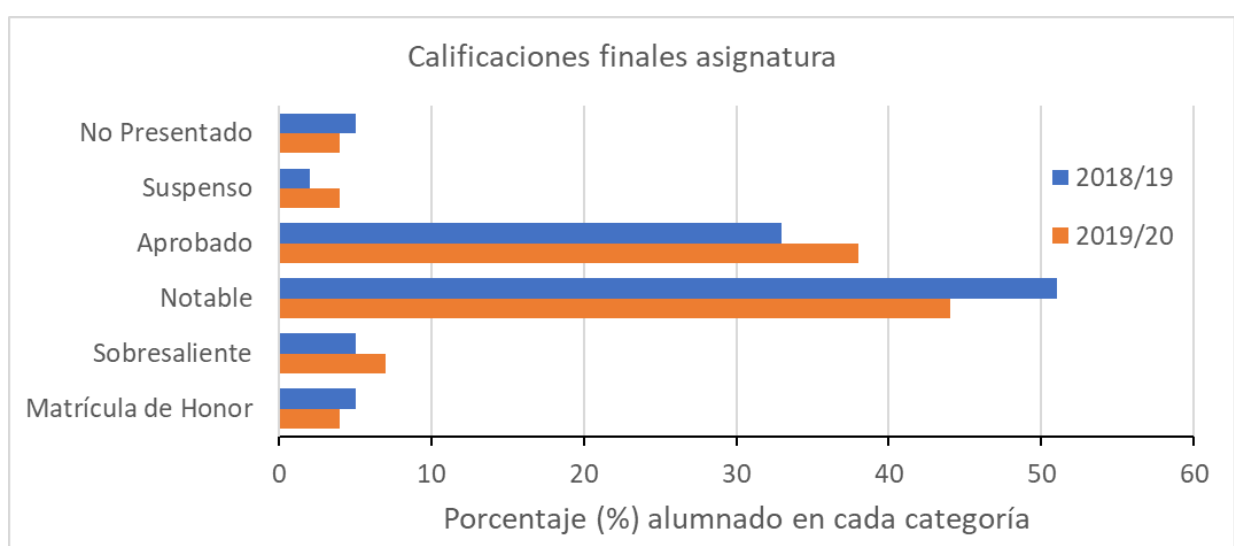

Figura 8. Comparativa del porcentaje de estudiantes que obtuvo cada calificación en la asignatura entre el curso 2018/19 (sin confinamiento, con prueba final presencial) y 21 2019/20 (con confinamiento, con prueba final en línea).

\section{Conclusiones}

El alumnado ha podido realizar el seguimiento en línea de la asignatura pese a la situación, observándose un cambio en el patrón de accesos al aula virtual de la asignatura durante el periodo de confinamiento, que se han incrementado en relación con lo observado el curso anterior.

La percepción de la medida en la que habían desarrollado las competencias y alcanzado los resultados de aprendizaje requeridos en la asignatura fue positiva, aunque varió entre determinadas competencias y objetivos.

Sus principales problemas percibidos para afrontar la evaluación en línea de la asignatura derivan de la posible pérdida de la conexión a Internet durante la prueba de evaluación, de los sistemas para realizar una comunicación síncrona durante el examen con el profesorado y de los problemas derivados de hacer el examen en un entorno no apropiado (conexiones de menor capacidad, distracciones familiares o ambientales). En menor medida, los derivados de un equipo informático no actualizado o de las posibles diferencies entre el diseño de la prueba para ser realizada presencialmente y en línea.
Las posibles soluciones planteadas por el alumnado en respuestas abiertas fueron no realizar el examen, sustituyéndolo por un trabajo o por las calificaciones de la evaluación continua, o añadir el suficiente tiempo para su entrega en la nueva situación. Comprobaciones previas del sistema o vías alternativas de entrega y comunicación, complementarias al campus virtual habitual, fueron propuestas como medidas para reducir su nerviosismo durante el examen.

Ninguna de las propuestas realizadas por el profesorado, dirigidas a facilitar una experiencia lo más similar posible al examen presencial y buscar vías complementarías a la entrega a través del campus virtual habitual y a la comunicación durante el examen fue considerada como una medida de mitigación adecuada para todo el alumnado. No obstante, sí que se observó que uniendo un número reducido de medidas sencillas la consideración acumulada de que se mitigaban bastante o casi completamente sus problemas percibidos alcanzó a entre el 81 y el $92 \%$ del alumnado.

Una vez implementadas, todo el alumnado fue capaz de realizar la prueba de evaluación online, obteniendo unas calificaciones globales en la asignatura similares a las del alumnado del curso anterior.

\section{Referencias}

Bargiela, I. M., Puig, B., \& Blanco Anaya, P. (2018). Las prácticas científicas en infantil: una aproximación al análisis del currículum y planes de formación del profesorado de Galicia. Enseñanza de las ciencias, 36(1), 7-23. https://doi.org/10.5565/rev/ensciencias.2311

Cabero Almenara, J., \& Ruiz Palmero, J. (2018). Las Tecnologías de la Información y Comunicación para la inclusión: reformulando la brecha digital. International Journal of Educational Research and Innovation, 9, 16-30.

Celik, V., \& Yesilyurt, E. (2013). Attitudes to technology, perceived computer selfefficacy and computer anxiety as predictors of computer supported education. $\begin{array}{llll}\text { Computers \& } \quad \text { Education, } & \text { 60(1), }\end{array}$ http://dx.doi.org/10.1016/j.compedu.2012.06.008

Chirumamilla, A., Sindre, G., \& Nguyen-Duc, A. (2020). Cheating in e-exams and paper exams: the perceptions of engineering students and teachers in Norway.
MAGISTER

Vol. 32. Núm. I: (2020). Sección extraordinaria 
Assessment \& Evaluation in Higher Education, in press. https://doi.org/10.1080/02602938.2020.1719975

de Pro Bueno, A., de Pro Chereguini, C., \& Doménech, J.C. (2019). ¿Cómo estamos formando a las futuras maestras para enseñar ciencias en el grado de educación infantil? Universitas Tarraconensis. Revista de Ciències de l'Educació, 2019.2, 88-99. DOI: https://doi.org/10.17345/ute.2019.2.2657

Doménech, J. C., de Pro Bueno, A., \& Solbes, J. (2016). ¿Qué ciencias se enseñan y cómo se hace en las aulas de educación infantil? La visión de los maestros en formación inicial. Enseñanza de las ciencias, 34(3), 25-50. Doi: http://dx.doi.org/10.5565/rev/ensciencias. 1870

Dorrego, E. (2016). Educación a distancia y evaluación del aprendizaje. $R E D$. Revista de educación a distancia, 50, art. 12. http://dx.doi.org/10.6018/red/50/12

Echeita, G. (2020). La Pandemia del Covid-19. ¿Una oportunidad para pensar en cómo hacer más inclusivos nuestros sistemas educativos? Revista Internacional de Educación para la Justicia Social, 9(1), 7-16.

Ferdig, R.E., Baumgartner, E., Hartshorne, R., Kaplan-Rakowski, R. \& Mouza, C. (2020). Teaching, Technology, and Teacher Education during the COVID-19 Pandemic: Stories from the Field. Waynesville: Association for the Advancement of Computing in Education (AACE). https://www.learntechlib.org/p/216903/

Fernández Manzanal, R., \& Bravo Tudela, M. (2015). Las ciencias de la naturaleza en la Educación Infantil. Madrid: Ediciones Pirámide.

García-Peñalvo, F.J., Corell, A., Abella-García, V. \& Grande, M. (2020). La evaluación online en la educación superior en tiempos de la COVID-19. Education in the Knowledge Society, 21, article 12 DOI: https://doi.org/10.14201/eks.23013

Gómez, D. C. (2019). Una aproximación a la evolución de la brecha digital entre la población joven en España (2006-2015). Revista Española de Sociología, 28(1), 27-44. Doi: http://dx.doi.org/10.22325/fes/res.2018.16

IESALC (2020). COVID-19 y educación superior: De los efectos inmediatos al día después. Análisis de impactos, respuestas políticas y recomendaciones. Caracas: Instituto Internacional para la Educación Superior en América Latina y el Caribe - UNESCO. https://www.iesalc.unesco.org/

Lozano-Díaz, A., Fernández-Prados, J. S., Figueredo Canosa, V., \& Martínez Martínez, A. M. (2020). Impactos del confinamiento por el COVID-19 entre universitarios: Satisfacción Vital, Resiliencia y Capital Social Online.
International Journal of Sociology of Education, 9(Extra 1), 79-104. http://doi.org/10.17583/rise.2020.5925

Mérida Serrano, R., Torres-Porras, J., \& Alcántara Manzanares, J. (eds.) (2017). Didáctica de las ciencias experimentales en educación infantil. Un enfoque práctico. Madrid: Editorial Síntesis.

Quijano López, R. (coord.) (2016) Enseñanza de las Ciencias de la Naturaleza en Educación Infantil. Madrid: Ediciones Pirámide.

Sanmartí, N. \& Machán Carvajal, I. (2015). La educación científica del siglo XXI: retos y propuestas. Investigación y Ciencia, 469, 31-39.

Smith, K. (2016). Functions of assessment in teacher education. En J. Loughran \& M.L. Hamilton (eds.) International handbook of teacher education (vol. 2, pp. 405-428). Singapur: Springer. https://doi.org/10.1007/978-981-10-0369-1_12

Torralba-Burrial, A., \& Herrero, M. (2018). Potenciando la inclusión mediante buenas prácticas en infoaccesibilidad: la Didáctica de las Ciencias de la Vida en la formación inicial de maestros de Educación Infantil y Primaria. En A.I. Allueva Pinilla \& J.L. Alejandre Marco (coords.) Casos de éxito en aprendizaje ubicuo y social mediado con tecnologías (pp. 109-117). Zaragoza: Prensas de la Universidad de Zaragoza.

*Autor de contacto: Antonio Torralba-Burrial, torralbaantonio@uniovi.es
MAGISTER

Vol. 32. Núm. I: (2020). Sección extraordinaria 